\title{
Reconfigurer la cartographie de la Franco- Amérique : la représentation de l'espace et du récit identitaire dans Nikolski de Nicolas Dickner
}

\author{
Jimmy Thibeault \\ Université de Moncton
}

Certains romans de la littérature québécoise ont proposé, particulièrement depuis les années 1980, une relecture des identités québécoise et canadienne-française à travers une ouverture des frontières identificatoires traditionnelles (géographiques, culturelles, nationales et linguistiques) et l'exploration de l'espace outre frontière que représente le 
continent américain ${ }^{1}$. Cette relecture de l'espace identificatoire, désormais ouvert à l'« ailleurs », partant à l'« autre », a entraîné dans son sillage une redéfinition de la notion même d'identité, qui tend de plus en plus à s'éloigner des repères traditionnels, liés à l'appartenance du soi à un « ici » et à un " nous », voire à un «même », identitaire, pour s'inscrire dans un rapport intime du soi au monde. Józef Kwaterko explique ce déplacement de la notion d'identité par le fait que

[la] société pensée, fondée sur les présupposées culturels et les idéologèmes de l'ici" et d'un "nous" homogène, se voit relayée par une société vécue, c'est-à-dire éprouvée au quotidien comme de plus en plus décalée des déterminations idéologiques et plongée dans l'hétérogénéité culturelle. (p. 147)

Dans cette "société vécue » que Kwaterko relève dans le contexte de la littérature québécoise, les identités ne se fondent plus sur les a priori de la naissance, mais se construisent plutôt à travers les expériences intimes que vit le sujet au fil de sa quête identitaire, et qui le situent dans un réseau d'identification se déployant à l'échelle continentale : c'est dans la pénétration de l'« ailleurs » et dans la rencontre de l'« autre » que le soi découvre les nouveaux repères qui lui permettront de progresser.

Le roman de la route offre un lieu propice à ce déplacement identitaire qui fait du sujet, du soi, le véritable centre du processus d'identification puisque « les protagonistes se transforment de sédentaires en nomades pour entreprendre un voyage identitaire qui les inspire à découvrir leur voie dans la vie » (Morency, den Toonder et Lintvelt, 2006, p. 7). En même temps que les protagonistes trouvent dans le voyage un espace

${ }^{1}$ La préparation de cet article a été rendue possible grâce à la subvention du CRSH accordée au projet Fictions de la Franco-Amérique. 
d'affirmation identitaire $\mathrm{du}$ soi, l'exploration du continent devient l'occasion de redécouvrir, de réinterpréter et de réactualiser les traces d'une présence collective qui dépasse les référents historiographiques et mythiques des discours traditionnels pour déboucher sur une expérience individuelle et multiple du continent. En effet, le voyage qu'entreprennent les protagonistes du roman de la route québécois se fait souvent sur les traces d'une certaine image de la Franco-Amérique ${ }^{2}$ qui hante la mémoire et l'imaginaire du continent. Cette image, qui renvoie d'abord à un discours historiographique et mythique usé par les anciennes idéologies nationales, est généralement réinterprétée par l'intermédiaire d'un discours de la marge, donc par un discours qui ne renvoie plus aux "grands récits » de l'Amérique, mais davantage aux «récits» multiples des individus qui ont parcouru le continent depuis son origine. C'est ainsi que Jacques Poulin, dans le roman phare de l'écriture de l'américanité québécoise que représente Volkswagen blues, revisite les grands mythes canadiens-français et américains en posant au centre du récit le discours de la marge par excellence, soit celui porteur de l'histoire oubliée de la figure de l'Amérindien. On comprend, au fil du roman de Poulin, que la relecture de l'espace ne peut plus se fonder sur la mémoire historiographique et muséale, marquée par l'oubli et le mensonge, mais qu'elle doit plutôt se faire dans la mise en valeur des expériences intimes telles que les ont vécues les premiers colons et que tend à réactualiser le regard métissé de Pitsémine. C'est dans cette relecture de l'expérience intime du continent que les protagonistes en viennent finalement à

2 Le terme de Franco-Amérique est emprunté aux travaux des géographes Dean Louder et Éric Waddell qui retracent, sur le continent américain, la mémoire et l'expression d'une Amérique qui se dit et se pense en français. 
affirmer leur identité individuelle, tout en repensant et en retraçant, à partir de la marge, une certaine cartographie identitaire de la Franco-Amérique.

Le présent article propose une lecture de cette cartographie de la Franco-Amérique telle qu'elle se présente dans le roman Nikolski de Nicolas Dickner. Ce roman récent s'inscrit effectivement dans le sillage des romans qui ont permis de repenser le rapport aux récits traditionnels et qui ont remis le sujet en avant-plan de la question de l'identification à un territoire qui serait de moins en moins marqué par la notion de frontières nationales. Aussi, bien qu'il ne s'agisse pas d'un roman de la route à proprement parler, retrouve-t-on au cœur même de la narration de Nikolski une conscience aiguë du continent qui, comme le constate Jean Morency, passe par « [1]'image de la diaspora acadienne et canadienne-française, épiphénomène de l'occupation française du continent nordaméricain » (2008, p. 37), image dont sont porteurs les trois personnages principaux du roman, le narrateur, Noah et Joyce. Morency remarque ailleurs, au sujet de la destinée canadiennefrançaise sur le continent, sans toutefois approfondir l'analyse du roman, que Nikolski

témoigne de façon éloquente de la survie de cet imaginaire particulier qui reste attaché à l'expérience continentale telle que vécue par les Canadiens français. Or, le roman de Dickner montre bien que cet imaginaire peut être convoqué de la façon la plus moderne qui soit, ce qui ouvre des perspectives nouvelles à propos de l'idée qu'on se fait du Canada français et de son possible réinvestissement symbolique. (2009, p. 163)

Mon propos portera moins, ici, sur la figure du « refoulé Canadien français », comme la nomme Jean Morency (2009), que sur la redéfinition de cet imaginaire dans l'inscription, par 
le parcours individuel des personnages, de la présence française en Amérique. Comment, à partir des expériences individuelles, Dickner reconfigure-t-il la cartographie de la Franco-Amérique? À partir de quelle(s) histoire(s) cette cartographie se construit-elle? Et comment, dans le contexte d'une "société vécue » par des individus, la Franco-Amérique, en tant qu'espace d'identification, peut-elle s'inscrire dans la cartographie du continent? J'analyserai d'abord les repères identificatoires spatiaux et mémoriels qui permettent aux personnages du roman de se définir, individuellement, dans l'espace continental. Ensuite, j'examinerai comment ces individualités affirmées sont réunies, par le discours narratif, dans la constitution d'un «nouveau grand récit » continental de la Franco-Amérique.

\section{Éclatement et réinterprétation des repères de la Franco- Amérique}

Définir l'espace de la Franco-Amérique en en traçant la carte n'est pas chose simple à faire, comme l'ont montré les géographes Dean Louder, Jean Morisset et Éric Waddell. Ceci pour plusieurs raisons historiques, sociales et politiques. D'abord, il importe de souligner qu'une bonne partie du caractère «Franco », pour reprendre l'expression des géographes cités,

a été systématiquement évincée de la Nord-Amérique avec le processus de recouvrement territorial et linguistique imposé depuis l'achat de la Louisiane et de l'Alaska par les États-Unis, et aussi la disparition du Nord-Ouest et du territoire de l'Orégon. (Morisset et Waddell, 2000, p. 14) 
De plus, plusieurs communautés et familles francophones éparpillées sur le continent ont longtemps survécu dans un relatif anonymat, en marge de l'histoire officielle de la région, de la province ou de l'état où elles sont implantées. Aussi, la voix de ces communautés, en plus de ne pas toujours trouver sa place dans le discours mémoriel de la majorité avec laquelle elles cohabitent, a-t-elle souvent été marginalisée au sein même de la francophonie alors que certaines collectivités plus imposantes, comme celle du Québec et de l'Acadie, ont pu «monopoliser» le discours identitaire de la présence francophone en Amérique. Il devient en conséquence difficile pour le géographe de retracer et de cartographier tous ces foyers qui constituent la Franco-Amérique. D’ailleurs, si cela était réalisable, cette cartographie serait constamment à revoir puisqu'elle subit les contrecoups du phénomène de la mondialisation alors que de nouveaux foyers francophones se fondent au gré d'une immigration de plus en plus importante dans les grands centres nord-américains :

En devenant une société ouverte et mondialisée, on peut avancer comme hypothèse que le Québec, et jusqu'à un certain point l'Acadie néo-brunswickoise, trouve de plus en plus ses interlocuteurs naturels chez les immigrants francophones et latinos des grandes villes de l'Amérique du Nord [...]. (Waddell et Louder, 2008, p. 22)

Ainsi, la cartographie de la Franco-Amérique est marquée par une «présence-absence » (ibid., p. 21) de ces communautés.

Comment alors tracer les contours de cet espace d'identification sinon en ayant recours à une autre forme de cartographie? Une cartographie qui tiendrait moins des traces historiques et géographiques laissées sur le continent que d'une mémoire et d'un imaginaire qui se construiraient en marge des 
"grands récits » traditionnels pour, comme le souligne Lucie Hotte au sujet de la littérature franco-ontarienne, « suppléer [à] une mémoire défaillante» (p. 362). Une construction de l'espace identitaire qu'assume d'ailleurs Michel Bouchard qui n'hésite pas à franchir les frontières symboliques de l'identité pour trouver, dans la mémoire du continent, des repères identificatoires qui lui seraient propres :

Nous sommes désemparés devant notre histoire. Elle est comme un rêve que l'on oublie en se réveillant le matin, mais qui nous tracasse. Nous savons qu'il y a des réalités refoulées dans notre inconscient, mais nous sommes incapables de nous en souvenir. Je sais que ma communauté francophone dépasse les frontières de mon village, ma province et même mon pays. Je revendique l'Amérique tout entière comme patrie. Je fais appel à mon histoire, l'histoire que je crée, pour justifier mon appartenance à ce continent. (p. 45)

Voici donc que se pointe, dans la reconfiguration de la cartographie franco-américaine, par l'éclatement des frontières institutionnelles et le dépassement du récit local, le début d'un récit global, qui est constitué, notent Waddell et Louder, « d'une part, de dimensions continentales et, d'autre part, de configuration pluraliste» (p.14). Un récit singulier, celui de Michel Bouchard, qui rejoint d'autres récits, ceux d'Éric Waddell, de Dean Louder, de Jean Morisset, de même que ceux que racontent les écrivains de cette Franco-Amérique, tous produits à l'échelle continentale, et dont la mise en réseau permettrait de retracer la cartographie d'une Franco-Amérique polyphonique. Un récit qui, dans le contexte postmoderne de dépassement des discours nationaux, se construit désormais autour de la notion de partage référentiel :

Le Québec se dénationalise! [...] les défis auxquels la FrancoAmérique fait présentement face ne proviennent pas de l'autre 
ou de l'ailleurs. Ils surgissent plutôt de l'intérieur, au sein d'une société extrêmement dynamique et enfin ouvertement pluraliste, une société qui possède la langue française en partage, qui est très sensible aux nombreuses manières d'habiter la Terre, mais qui épouse en même temps les valeurs d'un monde néolibéral, donc hautement individualiste et aveuglé par son propre pouvoir. Un paradoxe de plus! (Waddell et Louder, 2008, p. 22)

Un paradoxe continental qu'on retrouve particulièrement bien illustré dans la cartographie américaine que recompose Nicolas Dickner dans Nikolski.

\section{Le «paradigme absent » des cartes géographiques}

Le roman de Dickner participe effectivement à cette reconfiguration de l'espace franco-américain alors que la narration retrace, dans un chassé-croisé d'expériences continentales, dans un regard global sur le territoire identitaire, la cartographie d'une appartenance multiple, c'est-à-dire celle des protagonistes au territoire. Cette reconfiguration se fonde, à l'instar de ce qu'avance Michel Bouchard, sur la capacité des personnages (le narrateur, Noah et Joyce) à créer leur propre histoire et à justifier leur appartenance au continent, ce qui ne peut être possible qu'en se défaisant du cadre identitaire imposé par le milieu familial, qu'ils perçoivent comme un espace d'enfermement. Le narrateur, d'abord, pose, dès le début, son récit sous le signe de l'émancipation du soi par rapport à une existence passée sous l'emprise de sa mère, une Québécoise qui, dans sa jeunesse, a suivi le mouvement «beat » et a traversé le continent d'est en ouest, avant de se sédentariser en s'établissant dans une maison de banlieue. À la 
mort de cette dernière, le narrateur se sent libéré et entame un processus de détachement en mettant à la poubelle les souvenirs qu'elle avait accumulés au cours de sa vie - «pour réduire à néant cinq décennies d'existence, cinq placards de babioles écrasées sous leur propre poids» (Dickner, 2005, p. 12) - et en quittant la maison maternelle pour s'établir au cœur de la ville de Montréal :

Pareil ménage peut sembler sinistre et revanchard. Qu'on me comprenne bien : je me retrouvais soudain seul au monde, sans amis ni famille, avec l'urgente nécessité de continuer à vivre. (ibid.)

La rupture avec les anciens repères identificatoires, fournis par la présence de la mère, se précise davantage lors de la découverte d'une boussole un peu spéciale, ici dénommée « compas Nikolski », qui pointe toujours à $34^{\circ}$ à l'ouest du nord, faisant ainsi du village de Nikolski, dans les îles Aléoutiennes, plus précisément sur l'île Umnak, son véritable point de repère. Une déviation qui joue un rôle important dans le roman puisqu'elle permet de repenser l'espace identificatoire des personnages qui traversent la narration à partir de références à la fois singulières et collectives dans leurs marginalités. À l'instar du narrateur, Noah et Joyce dévient de leur trajectoire initiale afin de découvrir leur propre voie et d'affirmer leur propre voix dans la vie : Noah, d'origine acadienne et métisse, quitte la roulotte de sa mère, qui erre entre l'Ontario et les Rocheuses de l'Alberta3, pour se «fixer» à Montréal, pour

\footnotetext{
3 La mère de Noah, Sarah Riel, est décrite comme une femme des Plaines, descendante des Métis, qui ne peut sortir de l'espace contenu dans le seul univers qu'elle connaît, le centre du continent: «Le temps filait au rythme océanique de Grandpa. Rien ne semblait avoir changé, sinon la répartition de la rouille sur les flancs du Bonneville 1966. Sarah pilotait, Noah grandissait, et
} 
trouver ce «rassurant point fixe dans l'univers» (p. 107) auquel s'identifier. Joyce, pour sa part, fuit son village de Tête-àla-Baleine, sur une île de la Basse-Côte-Nord, et, surtout, la famille envahissante de son père ${ }^{4}$, pour suivre les traces de ses ancêtres maternels en devenant pirate, à Montréal. Ces personnages, bien qu'ils suivent des parcours différents, sont liés par des liens qui apparaissent au fil du roman (rencontres, amitiés, espace d'habitation, etc.), mais dont le plus important est celui d'appartenir, sans le savoir, à la même famille acadienne, les Doucet, qui a immigré à Tête-à-la-Baleine peu avant la déportation de 1755 - le narrateur et Noah sont tous les deux les fils de Jonas Doucet, un bourlingueur de Tête-à-laBaleine et oncle mythique de Joyce.

Cette appartenance, hautement symbolique, à un peuple qui se définit essentiellement par le fait qu'il n'a pas de territoire identitaire à lui (à laquelle Noah peut ajouter son appartenance aux nations métisse et autochtone héritée de sa mère), explique l'urgence que ressentent les personnages de reconfigurer la cartographie de leur présence au monde et, ce faisant, la mémoire des espaces identificatoires auxquels ils tentent de se rattacher. On le constate notamment par la présence de cartes géographiques - cartes routières, cartes marines, cartes nationales et régionales ou cartes

la roulotte semblait toujours frappée par une malédiction circulaire.» (Dickner, 2005, p. 45)

4 « Le père de Joyce n'ayant jamais voulu se remarier, cette dernière demeura orpheline et fille unique, seule maître à bord après Dieu - bref, chargée de préparer les repas, torcher la maison et faire ses devoirs toute seule, tâches dont elle s'acquittait déjà de façon routinière à l'âge de six ans [...] Mais le plus ardue de toutes ses tâches consistait à supporter la famille de son père, assortiment de tantes inquisitrices, de cousins turbulents et d'oncles tapageurs qui débarquaient à la moindre occasion. » (Dickner, 2005, p. 56-57) 
touristiques - qui parsèment le roman et qui se multiplient, sans toutefois saisir complètement la profondeur des lieux, à mesure que les personnages semblent chercher leurs repères. Tout se passe comme si ces cartes étaient incomplètes puisqu'elles ne renvoient à aucune signification permettant de saisir l'espace, et que la réalité qu'elles tentent de représenter se trouvait en marge de la représentation officielle du continent. Ainsi, le paradigme des cartes semble vidé de sens, sens que les personnages, rattachés d'abord à des lieux clos, doivent découvrir et intégrer à leur imaginaire propre en en prenant physiquement la mesure. Confrontés à cette incomplétude des cartes, Noah et Joyce - à qui le narrateur cède rapidement la place - s'engagent, chacun de leur côté, dans un parcours qui leur permet de découvrir, de s'approprier et de redéfinir, à travers les expériences individuelles, une cartographie qui leur serait propre.

Dès l'enfance, les cartes géographiques jouent un rôle clef pour Noah et Joyce: Noah, qui passe la sienne à errer sur la plaine canadienne, apprend à lire sur les cartes routières, ce qui l'amène à se construire un imaginaire identitaire qui ne se fonde pas sur le récit historique des lieux, mais sur la sonorité des noms de villes, de villages, de parcs, de lacs et de routes qu'il ne peut observer que de l'extérieur, sans jamais pouvoir les intégrer. Pour Joyce, qui se sent prisonnière de la maison et du cercle familiaux, les cartes marines de son père représentent un espace de liberté qui lui permet de fuir sa réalité d'insulaire. Les deux personnages trouvent ainsi dans les cartes géographiques un point de fuite vers une liberté imaginaire, comme si ces cartes permettaient d'abolir les frontières et de surpasser leur point d'horizon. On le constate d'ailleurs lorsque 
Joyce en arrive à percevoir différemment la clôture de l'école selon l'endroit où elle se tient :

Douze heures plus tôt, elle était encore prisonnière de cet enclos; maintenant, l'endroit lui paraît totalement étranger [...] Il faut dire que l'apparence d'une clôture change considérablement selon le côté où l'on se trouve. Or, de ce côtéci, le grillage n'évoque que l'inoffensif quadrillage d'une carte géographique. (p. 53)

Cette impression de liberté, hors des frontières clôturées qu'abolit l'image de la carte géographique, correspond à celle qu'elle ressent lorsque, dès son jeune âge, elle se laisse absorber par les cartes qu'elle découvre dans le bureau de son père et, plus particulièrement, par «la carte 2472-B, une immense projection à l'échelle 1:100 000 du littoral de la BasseCôte-Nord avec, en plein milieu, le minuscule village de Tête-àla-Baleine» (p.54). La représentation topographique de l'espace habité semble donc procurer aux personnages une liberté qu'ils ne peuvent atteindre autrement.

À cet imaginaire des cartes, s'ajoute celui d'un autre type de cartographie : celui retracé par le récit de voyage dans les mers des Caraïbes que le grand-père de Joyce, dernier représentant de la famille Doucet sur l'île - la mère de Joyce est donnée pour morte par la famille du père - , intègre à la mémoire familiale par l'histoire des pirates Herménégilde et Alonzo Doucette ${ }^{5}$. Un récit qui façonne l'identité de Joyce qui, à la mort du grand-père, se précise davantage dans le sillage de ses ancêtres :

\footnotetext{
5 Cette branche de la famille Doucet se démarque cependant de celle de Joyce par la graphie, comme si chaque destinée devait attribuer au sujet un nom propre à sa condition. Aussi Joyce retrouvera-t-elle dans la cartographie américaine des traces de différentes branches de la famille Doucet à la graphie tout aussi variée que leur parcours continental.
} 
Joyce était donc la dernière Doucet du village. Digne descendante de ses aïeux, elle avait développé un caractère solitaire qui donnait à son visage une maturité précoce et inquiétante [...] Elle souffrait par ailleurs de claustrophobie, un problème sans doute naturel lorsqu'on appartient à une famille éparpillée aux quatre coins de l'Amérique du Nord. (p. 62)

Comme Joyce, Noah est également en contact avec des récits de piraterie se déroulant dans les mers des Caraïbes, lorsqu'il découvre un livre sans titre qu'a laissé son père, Jonas Doucet, lors de son unique passage dans la roulotte :

C'était une sorte de recueil d'histoires de marins dont la première page reproduisait une carte des Caraïbes qui ne cessait de stupéfier Noah. Comment pouvaient coexister une telle masse d'eau et une si petite quantité de terre? La carte de la Saskatchewan ressemblait à un négatif des Caraïbes : pour chaque île, un lac, et des océans de graminées en guise de mer. (p.38)

Cet espace livresque - qui appartient, dans une certaine mesure, au même imaginaire familial que Joyce - s'amalgame à celui qu'il retrouve sur les cartes routières et dans les récits familiaux de la mère pour finalement participer à la formation de son identité : «jamais plus il ne saurait distinguer un livre d'une carte routière, une carte routière de son arbre généalogique, et son arbre généalogique de l'odeur de l'huile à transmission. » (p. 39) Joyce se reconnaît également dans une certaine comparaison de son univers local à l'espace continental lorsqu'elle retrouve dans son environnement familial une île de même nom que celle qu'on avait surnommée l'île aux pirates, c'est-à-dire «le mythique repaire de l'île Providence » :

Ce toponyme déconcerta Joyce : elle passait chaque été sur l'île Providence et n'y avait jamais remarqué le moindre repaire de 
pirates, que de vieilles maisons de bardeaux peuplées d'oncles et de cousins tapageurs.

Lyzandre Doucet expliqua qu'il s'agissait d'une autre île Providence, située au nord de l'île Hispaniola, dans les Caraïbes [...] les pirates avaient fait de cette île un repaire imprenable, où ils ne craignaient personne. Ils occupaient un havre à deux embouchures, facile à protéger, dont la faible profondeur interdisait l'accès aux obèses vaisseaux militaires - ce qui, du point de vue de Joyce, impliquait ni oncles ni cousins, et prouvait sans l'ombre d'un doute qu'il s'agissait d'une tout autre île. (p. 63)

On constate cependant que, s'il y a reconnaissance dans les cartes géographiques, les espaces cartographiés sont également criblés de vides que les personnages sont amenés à remplir lorsqu'ils affirment leur volonté de prendre pied dans l'espace réel, qui leur semble jusque-là inaccessible.

Ces vides s'imposent aux deux personnages alors qu'ils tentent de donner une profondeur aux espaces nommés par les cartes: ces noms de lieux sur les cartes géographiques apparaissent, en quelque sorte, comme des «paradigmes absents » - pour reprendre l'expression de Marc Angenot (1978) —, c'est-à-dire comme des noms ne renvoyant à aucune réalité connue et dont les personnages sont obligés d'inventer le sens. C'est d'ailleurs ce que Noah constate alors qu'il rêve de s'installer dans une des villes qu'il traverse, d'aller à l'école et de fréquenter des enfants de son âge :

Il ne partageait pas le Glorieux Imaginaire Routier NordAméricain. De son point de vue, la route n'était rien qu'un étroit nulle-part, bordé à bâbord et tribord par le monde réel endroit fascinant, inaccessible et inimaginable. (p. 46)

Comment, en effet, donner un sens à un espace dont il ne peut que rêver le mode de vie? Un problème que posent notamment les noms de lieux qu'énonce le père de Noah au fil 
de sa correspondance avec la mère : si Noah parvient à tracer la cartographie des errances de Jonas depuis la ColombieBritannique jusqu'au village de Nikolski, d'où provient sa dernière carte postale, le sens de ces noms de lieux n'en demeure pas moins inaccessible; d'autant plus que la communication avec Jonas est incertaine puisqu'elle passe par un réseau de communications fondé sur le hasard, car ils sont toujours, chacun de leur côté, en mouvement sur le continent 6 , et que l'écriture de Jonas est souvent illisible. Noah s'explique le silence de son père en ayant recours à son imaginaire :

Les yeux rivés sur la ligne d'horizon, il ruminait des kilomètres d'idées noires en tentant d'imaginer ce que Jonas pouvait bien manigancer à Nikolski. Sans doute s'était-il amouraché d'une Aléoute et travaillait-il à se refaire une nouvelle vie en biffant toutes les tentatives antérieures. Noah imaginait un troupeau de demi-frères et de demi-sœurs aux yeux bridés, petits sédentaires crottés qui auraient monopolisé l'attention de son père. (p. 43-44)

Joyce vit sensiblement le même rapport à l'incomplétude que Noah, comme en témoignent les cartes marines de son père. D’une part, ces cartes ne lui permettent pas de sortir de sa réalité quotidienne au village, de se dégager de ses obligations à l'égard de son père, de ses oncles, de ses tantes et de ses

\footnotetext{
6 « Lorsque venait le temps de poster une lettre, elle [la mère] dépliait les cartes routières de l'ouest du continent sur le capot de Grandpa et tentait de deviner où Jonas pouvait bien se trouver. Par exemple, s'il venait de passer quelques semaines à Whitehorse, elle pensait le débusquer à Carmacks. Elle changeait ensuite d'avis : Carmacks était situé trop loin de la mer. Jonas avait plutôt continué sur la route 1 en direction d'Anchorage et il se trouvait sans doute quelque part à mi-chemin. Elle adressait donc la lettre à la poste restante de Slana et indiquait, en guise d'adresse de retour, la poste restante d'Assiniboia, où elle prévoyait passer au cours des semaines suivantes. [...] ils parvenaient à échanger, bon an mal an, une lettre par mois. » (Dickner, 2005, p. 39-40)
} 
cousins. D'autre part, elle remarque que la cartographie dont elle dispose ne représente que la mer, comme si le continent restait à cartographier : "Si les îles abondaient sur les cartes marines de la région, on notait en revanche une flagrante absence de route. » (p.55) Or, Joyce apprend, grâce à son grand-père, que sa mère n'est pas morte, mais qu'elle s'est enfuie quelques mois après sa naissance vers le continent: «Certains prétendaient qu'elle était allée à Montréal, voire aux États-Unis. » (p.68) Comme Noah qui entretient un rapport trouble avec son père, Joyce est aux prises avec l'épineux problème de comprendre le parcours de sa mère à travers une représentation incomplète du monde. Face à un monde certes cartographié mais dépouillé de sens, de profondeur, Noah et Joyce se trouvent donc confrontés à un vif besoin de quitter le lieu restreint de la roulotte, pour le premier, et du village de Tête-à-la-Baleine, pour la seconde, afin de construire leur propre représentation du réel et, finalement, d'y intégrer leur propre destinée.

Le départ de leur lieu d'origine ne représente pas une rupture nette avec l'espace d'identification imposé dans l'enfance, bien que Noah conçoive sa candidature dans une institution universitaire comme un "plan d'évasion » et que Joyce fugue littéralement de la maison, car, malgré la distance, ils conservent des liens avec la maison : Noah entretient une correspondance avec sa mère, sur le même modèle que celle qu'elle avait avec Jonas Doucet, et Joyce n'habite pleinement l'espace montréalais que lorsqu'elle retrouve, à la poissonnerie Shanahan, les odeurs familières de la mer $^{7}$. La fuite devient

\footnotetext{
7 Alors qu'elle ne trouve aucun repère auquel se rattacher, Joyce s'approche de la poissonnerie Shanahan : "L'odeur est si familière que Joyce sent des larmes lui monter aux yeux. » (Dickner, 2005, p. 83) Aussi, après avoir accepté
} 
ainsi, pour les personnages, un processus d'affirmation d'un soi qui tente d'acquérir la liberté de s'inscrire, individuellement, dans la géographie du continent en y traçant leur propre trajectoire. Aussi, lorsque vient le temps de partir, les protagonistes se tournent-ils vers une destination qui, parce qu'ils n'ont pas accès à sa représentation géographique, est restée vierge dans leur imaginaire. Noah explique d'ailleurs son choix de se rendre à Montréal, plutôt que de fréquenter une université à Winnipeg ou à Saskatoon, par son désir de « sortir de la boîte à gants, sauter par-dessus l'horizon » (p. 47). L'ouest ne convient pas davantage puisqu'il est déjà trop chargé par l'image qu'il s'en était faite, d'une part, à travers sa lecture des cartes routières et, d'autre part, par la figure d'un père inaccessible et en disgrâce :

L'ouest? Il était percé de toutes parts, transparent et graisseux comme les cartes routières de la boîte à gants. L'ouest, c'était son père, homme lointain et mystérieux qui habitait avec une tribu d'Aléoutes sur une île perdue de la mer de Béring, se nourrissait de saumon cru et chauffait sa yourte avec des bouses de mouton séchées - modèle paternel peu édifiant. (p. 47)

Il ne lui reste, finalement, qu'à emprunter le chemin inverse des pionniers qui ont repoussé les frontières de l'ouest et à prendre la route de l'est, plus précisément de Montréal, espace qui reste, pour lui, à découvrir. Joyce, comme Noah, inscrit sa fuite sous le signe de la découverte d'un nouveau

un emploi à la poissonnerie, Joyce trouve un certain réconfort qui lui permettra, ensuite, d'habiter la ville: «Joyce ressort de la poissonnerie Shanahan avec la consigne de revenir le lendemain à neuf heures pile. Elle traverse la rue puis, après s'être assurée que personne ne la regarde, renifle l'odeur de sang qui imprègne sa paume. En fermant les yeux, elle se croirait presque revenue dans la cuisine de son père, à Tête-à-la-Baleine. » (p. 86) 
territoire auquel s'identifier alors qu'elle arrive sur le continent, à Sept-Îles, en exploratrice et en cartographe :

Elle sortait enfin des cartes marines de son père pour s'aventurer dans un monde non cartographié, sans doute grouillant de périls inconnus, mais où l'on pouvait prendre toutes les routes que l'on désirait. (p. 71)

Certes, elle découvre qu'il existe déjà une cartographie du territoire - qui, de Sept-îles, se limite à la route 138 - , mais, pour elle, le territoire reste à inventer en ce sens qu'elle doit donner une profondeur aux paradigmes qui se cachent derrière une toponymie qui dépasse les limites de son imagination. Joyce et Noah devront cependant réhabiliter, d'une certaine manière, l'expérience de l'origine, depuis leurs ancêtres, afin de trouver leur place dans le nouvel espace à habiter.

\section{Élargir la cartographie identitaire au continent}

Dans Nikolski, Montréal devient le lieu d'une affirmation identitaire possible dans la mesure où les protagonistes se dégagent des contraintes imposées à la naissance, tout en se réconciliant avec l'histoire familiale. Dans l'espace de l'enfance, Noah et Joyce sont effectivement confrontés à un malaise identitaire, car ils ont l'impression de mener une vie autre, en marge de la réalité et, surtout, de soi. L'errance de Noah, par exemple, s'explique en partie par les histoires que sa mère lui raconte au sujet de ses ancêtres qui ont perdu leurs droits territoriaux lorsque les Blancs les ont enfermés dans des réserves gouvernementales. Les récits de Sarah, empreints d'une nostalgie de nomade, appellent constamment la figure 
des ancêtres qui ne trouvent aucun lieu de repos et qui hantent la roulotte familiale :

Deux Indiens chipeweyans sont assis à la table de la cuisine [...] Le premier est [1']arrière-arrière-grand-père [de Noah]. On ne sait rien sur eux, sinon qu'ils ont vécu et sont morts dans le nord du Manitoba à la fin du dix-neuvième siècle. (p. 27)

De manière plus pragmatique, l'errance de la mère s'explique également par le fait qu'elle a perdu, vis-à-vis des institutions, " son statut d'Indienne et le droit d'habiter sur une réserve » (p. 29) lorsqu'elle a épousé un Blanc. Une "subtilité administrative » qui n'est pas sans importance puisque le lien la rattachant au monde des Blancs se rompt

dix mois après les noces, lorsque Sarah décampa du domicile conjugal avec un œil au beurre noir, un sac de vidange hâtivement rempli de vêtements et la ferme intention de ne plus revenir en arrière. (p. 29)

À cette errance de la mère, s'ajoute celle du père, de Jonas Doucet, qui, par ses origines acadiennes, lègue également à Noah une identité qui n'a aucun territoire d'appartenance, au sens géopolitique du terme. C'est dans cette double absence de territoire identitaire que Noah, enfant, affirme sa première identité : « Noah aimait le contraste entre les deux versants de sa généalogie, le paradoxe d'être à la fois descendant des réserves et de la déportation. » (p. 30) Noah ressent cependant un malaise à l'égard d'une telle définition identitaire puisqu'il n'arrive pas à saisir nettement ce territoire auquel il appartient, comme lorsqu'il aperçoit une voiture sur la route alors qu'il tente d'uriner dans un fossé d'irrigation :

En retournant vers la roulotte, il médite sur cette étrange pudeur. Il ne peut chasser l'impression désagréable que ce véhicule empiétait sur son territoire intime, comme si la route 627 traversait en fait leur salle de bain. (p. 28) 
Ce à quoi le narrateur ajoute: «À bien y penser, cette image n'est pas très loin de la réalité.» (p. 28) Si Noah, en raison de l'histoire familiale, semble condamné à errer en marge des espaces sociaux rencontrés dans la plaine canadienne, Joyce ne parvient pas davantage à intégrer le monde social qui l'entoure alors qu'elle ne trouve jamais vraiment sa place au sein de la communauté de Tête-à-laBaleine et, plus particulièrement, au sein de la famille de son père. Cette exclusion découle essentiellement de la perception qu'a l'ensemble du village de la famille Doucet, dont les ancêtres pirates ont forgé de longue date, dans la mémoire collective, une image de marginaux :

Dans cette maison [qu'habite le grand-père de Joyce] étaient nés et avaient vécu tous les Doucet de Tête-à-la-Baleine : grandpère et grand-mère, grands-oncles, grands-tantes, cousines, cousins, beaux-frères et chiens galeux. Cette branche de la famille avait cessé de pratiquer la piraterie sans pour autant avoir développé la vocation de la pêche. Cette absence de rôle précis avait contribué à les isoler du reste de la population. (p. 60-61)

Cette marginalisation, que tend d'ailleurs à confirmer la localisation, hors du village, de la maison — « De toute façon, les Doucet habitaient trop loin du village pour ne pas être louches. » (p. 61) —, semble d'emblée exclure Joyce des jeux de ses cousins : "On ne demandait rien à cette petite cousine bizarre et, pour tout dire, on s'apercevait à peine de sa présence. » (p. 65) Pour se défaire de ce malaise qui les habite, Noah et Joyce fuient donc l'espace familial et intègrent celui de Montréal, dont la cartographie qu'ils traceront, chacun à leur manière, se pose sous le signe d'une ouverture sur le continent. C'est dans ce passage vers Montréal qu'il devient possible pour Noah et Joyce de trouver leur propre voie/x et d'accorder, par 
la réactualisation d'une mémoire oubliée des origines, leur destinée individuelle avec celle, globale, des Amériques.

La quête des personnages, si elle les oblige à rompre, du moins en partie, avec le milieu familial, les amène également à repenser leur appartenance au monde par une réactualisation de l'histoire familiale ancienne, c'est-à-dire celle dont la trace reste profondément gravée dans l'imaginaire continental. Ce retour obligatoire à l'origine est symbolisé, chez Noah, par son désir d'étudier l'archéologie à l'université et, plus particulièrement, la préhistoire amérindienne, un choix qui s'impose d'ailleurs alors qu'il sombre dans le sommeil, tête première dans le guide des programmes universitaires :

Il émergea une heure plus tard, nauséeux [...] La bouilloire lui renvoya l'image difforme de son visage. Au beau milieu du front, l'encre bon marché avait imprimé un mot mystérieux : Archéologie. (p. 49)

Pour Joyce, l'histoire familiale de la mère fait déjà partie de son identité par le récit que lui fait son grand-père des aventures de ses ancêtres pirates sur le continent américain :

En cédant l'Acadie aux Anglois, Louis XIV plongea tous les colons dans une situation délicate, en particulier la famille Doucet dont on n'avait pas oublié les raids sur la NouvelleAngleterre. Voyant s'approcher l'orage, les enfants d'Herménégilde devancèrent la déportation et s'éparpillèrent dans toutes les directions, de la Baie des Chaleurs au golfe du Mexique. L'errance et l'incertitude politique remirent la flibuste à l'ordre du jour. (p. 59)

Un récit familial qui a non seulement été influencé par les événements historiques du continent, mais qui s'inscrit également dans cette histoire continentale alors que le grandpère souligne que « Jean Lafitte, le légendaire pirate louisianais, aurait été un lointain petit-cousin» (p.60). La légende des 
Doucet amène ainsi la jeune Joyce à affirmer son « ambition de perpétuer les traditions familiales » (p. 63) et, par le fait même, à refuser d'entrevoir son avenir dans le lieu clos du village :

Il lui semblait inconvenant que l'arrière-arrière-petite-fille d'Herménégilde Doucette consacrât sa vie à éviscérer des morues et faires ses devoirs de sciences naturelles. Elle était destinée à devenir pirate, morbleu! (p. 63-64)

Il y a donc, dans le parcours des deux protagonistes, un processus de réappropriation, conscient ou non, de l'histoire familiale ancienne qui leur permet de se définir soi-même, au présent, dans l'espace identitaire que représente le continent. Il semble cependant que ce processus ne peut se réaliser qu'en marge des espaces définitoires traditionnels, de la famille, certes, mais également du cadre, voire de l'ordre, institutionnel.

La route que suit Noah vers une réappropriation de l'espace identitaire se pose, dès son arrivée à Montréal, sous le signe d'une illégitimité institutionnelle. D’une part, Maelo, le colocataire de Noah, accepte de lui louer une chambre parce qu'il reconnaît en lui un "réfugié politique », donc un individu coupé de sa terre d'origine, ce qui est d'ailleurs une condition à la location de la chambre : «À PARTAGER 41/2Petite Italie nfumeur pas d'animaux libre maintenant. Priorité aux réfugiés politiques. Appeler Poissonnerie Shanahan demander Maelo. » (p. 92) Cette représentation de Noah en réfugié politique est évidemment symbolique, mais n'en reflète pas moins le rapport trouble qu'il entretient avec l'espace identitaire: "Pour tout dire, il se sent indigne d'occuper cet endroit, comme s'il craignait de gaspiller quelque chose.» (p.95) Noah se demande : «Peut-on gaspiller du vide?» (p. 96) Si la question de Noah concerne surtout la chambre que lui loue Maelo, elle s'applique également à l'ensemble du territoire qu'il a parcouru 
et qui, pour lui, reste vide de sens. Un vide qu'on peut attribuer à sa double origine parentale ${ }^{8}$, mais surtout à son origine amérindienne qui semble exclue de tout espace d'appartenance. Son identité amérindienne problématique gagne d'ailleurs en importance lorsqu'il est confronté, dans un cours universitaire, à un discours essentiellement fondé sur une série de clichés bien ancrés dans l'imaginaire collectif dans lesquels il ne se reconnaît pas :

Edmond Scott sortait tout droit du dix-neuvième siècle - les mauvaises langues prétendaient même qu'il avait personnellement connu le grand chef Sitting Bull. Assis devant un auditorium presque vide, il donnait imperturbablement le même cours depuis 1969, présentant le panorama des Algonquins, des Sioux et des Nootkas comme une collection de poissons morts flottant dans des jarres d'esprit-de-vin. (p. 133)

Si le discours de Scott ne correspond en rien à l'expérience de Noah, ce dernier ne se sent pourtant pas justifié à rectifier les dires du professeur. Comment, en effet, expliquer une perception du monde fondée sur un «savoir approximatif et rapiécé, qui sentait le foin et l'huile à moteur, [et qui] ne trouvait pas sa place dans une salle de classe » (p.134)? Le directeur de thèse de Noah, le professeur Thomas SaintLaurent, qui se spécialise dans l'archéologie des déchets, confirme d'ailleurs cette impression en suggérant à son étudiant, qui propose comme sujet de mémoire de maîtrise « une étude comparative entre le développement du système routier et l'expansion des dépotoirs au cours des années 70 » (p.136), de choisir plutôt un projet de recherche qui cadre mieux avec le discours conservateur de l'université. Noah se

8 Les deux nations que Noah porte en lui sont d'ailleurs associées lorsqu'il compare le déplacement d'un village inuit à la déportation acadienne (p. 148). 
tourne finalement vers l'étude de la préhistoire amérindienne - autant dire de sa propre histoire.

Le travail universitaire n'offre cependant pas de réponse satisfaisante à la quête de Noah et il en vient à se demander s'il n'avait pas «quitté la Saskatchewan et parcouru plusieurs milliers de kilomètres afin d'étudier l'une des disciplines les plus ennuyantes au monde» (p.133). En fait, le véritable apprentissage de Noah, du moins sous l'angle de la problématique identitaire, se fait en marge des espaces officiels alors qu'il est amené, avec l'aide d'immigrés sud-américains, à tracer sa propre cartographie de la ville, d'abord, et celle du continent, ensuite. Cette cartographie tracée par Noah se démarque des cartes préexistantes en ce qu'il parvient à lui donner une profondeur culturelle que les autres ne possèdent pas. Il dresse ainsi une carte de la ville qui porte non seulement le nom des rues et des lieux, mais également toute la diversité des accents qu'on y trouve :

Au guidon du vélo, il construit une vue aérienne du territoire places, ruelles, murs, graffitis, cours d'école, escaliers, bazars et casse-croûte - tandis que, en discutant avec les clients, il espionne les accents, les vêtements, les traits morphologiques, les odeurs de cuisine et les bribes de musique. Additionnés l'un à l'autre, les deux inventaires composent une carte complexe du quartier, à la fois physique et culturelle. (p. 115)

Montréal se définit donc à la fois comme un point de rencontre de différentes cultures et comme une porte d'entrée sur le continent. Pourtant, malgré cette carte culturelle de la ville, Noah n'arrive pas à habiter complètement le continent, du moins jusqu'à sa rencontre avec Arizna, une jeune Vénézuélienne. 
C'est effectivement en fréquentant Arizna, qui travaille à la revendication des droits territoriaux pour les Amérindiens, tant du nord que du sud du continent, que Noah est amené à modifier la perception qu'il a de sa propre identité amérindienne et qu'il parvient, en réhabilitant cette dernière, à trouver sa véritable place dans l'espace continental. L'idée de son identité amérindienne est d'abord floue et Noah ne comprend pas comment elle pourrait lui permettre d'intégrer le territoire et de mettre fin au malaise qu'il ressent depuis l'enfance. On le constate clairement dans l'extrait suivant où, lors de sa première rencontre avec Arizna, il remet en question le droit des Autochtones à se réclamer d'un espace traditionnel qui, de son point de vue, n'en est pas un :

Attends un peu... Avant l'arrivée des Blancs, les Inuit se déplaçaient en fonction du gibier et des saisons. Les villages actuels se sont à peu près tous agglutinés autour des comptoirs de la compagnie de la Baie d'Hudson. Ce qui fait qu'au moment de leur relocalisation, les familles d'Inukjuak étaient sédentarisées depuis à peine deux ou trois générations. Alors est-ce qu'on peut encore parler de territoire traditionnel lorsque la manière d'occuper le territoire n'a plus rien de traditionnel? (p. 149)

La perception qu'a Noah de la situation territoriale des Amérindiens suit donc une logique de perte, de dépossession et d'effacement identitaire, car il ne tient compte que des histoires que lui racontait sa mère, sans replacer la question amérindienne dans son contexte global. Ce qu'apporte Arizna, en fait, c'est cette vision globale qui fait qu'un « territoire ne se mesure pas en kilomètre carrés » (p. 149), qu'il est chargé d'une histoire à travers laquelle les peuples s'affirment ou meurent :

Tu dois aussi considérer les ancêtres, la postérité, la tradition orale, les pistes de ski-doo, les liens familiaux, la chasse au 
phoque et la pêche au saumon, le lichen, les recours juridiques contre Hydro-Québec... le territoire, c'est surtout l'identité. (p. 149-150)

Ainsi, le rapport entre le territoire identitaire et la problématique des Amérindiens se trouve, à travers le discours d'Arizna, élargi à un point tel qu'il n'est plus question d'une représentation du monde en termes de frontières municipales ou communautaires, mais plutôt en termes d'un espace continental à la temporalité flottante. On retrouve, dans cette abolition des frontières nationales et temporelles, une remise en question des "grands récits» qui structurent le discours officiel, ce qui représente un passage obligé dans l'affirmation des identités amérindiennes. C'est pourquoi, notamment, elle étudie dans une université non officielle de Caracas, qu'elle rompt avec les différenciations traditionnelles entre les Amérindiens du nord et ceux du sud et qu'elle met sur pied, à la fin du roman, une maison d'édition consacrée aux études indigénistes. Bien qu'il ne s'implique pas directement dans les projets d'Arizna et qu'il ne participe pas à ses travaux, Noah apprend, à son contact, mais également de son fils Simón (qui est aussi celui de Noah), à affirmer et à assumer sa place dans l'espace continental. Ce processus identitaire se précise alors qu'il rentre à Montréal avec Simón et qu'il passe les douanes en n'ayant en sa possession qu'une simple lettre écrite par Arizna, où elle confirme sa paternité, pour expliquer la présence du garçon à ses côtés dans ce voyage de retour à travers les Amériques :

À l'aéroport de Newark, les formalités douanières se déroulent sans anicroche. L'arrivée simultanée d'un père à moitié chipeweyan et d'un fiston à moitié vénézuélien ne semble pas étonner le douanier. (p. 302) 
La facilité avec laquelle les deux voyageurs passent les douanes a de quoi surprendre, mais on comprend que cette frontière qu'ils traversent ne les concerne pas, qu'elle ne soit qu'une formalité sans importance pour eux dont la demeure se trouve partout sur le continent. L'arrivée à Montréal, chez Maelo, ne semble déstabiliser ni le père, ni le fils, ni Maelo qui les accueille comme s'ils étaient de sa famille et qui offre à Noah de reprendre son ancienne chambre. C'est à ce moment, dans cette chambre que Noah percevait au début du roman comme un espace immense et vide, qu'il constate que l'espace est désormais porteur de sens, d'une mémoire et d'un imaginaire qui lui est propre et qu'il peut léguer à son fils. Dès lors, Noah ne ressent plus le malaise du début à l'égard de l'espace qui l'entoure. À travers les liens qui l'unissent à Arizna, à Simón et à Maelo, il apprend à habiter un espace auquel il peut désormais s'identifier.

Si Noah trouve sa place dans l'espace américain à travers les différentes rencontres qui l'amènent de la roulotte maternelle à Montréal, en passant par le Venezuela, et qui lui permettent d'affirmer sa présence sur le continent, Joyce, pour sa part, connaît d'emblée la voie qu'elle veut suivre. Ce qui est moins évident pour elle, c'est de savoir comment elle peut y parvenir. En effet, comment devenir pirate à la fin du XX ${ }^{\mathrm{e}}$ siècle? La solution se trouve évidemment dans l'histoire légendaire des ancêtres pirates qui transgressaient les frontières et les lois, mais surtout dans la réhabilitation d'une histoire plus récente. Cette réhabilitation passe d'abord par les cartes postales envoyées par l'oncle Jonas, qui est le premier à fuir le village 
pour embrasser le monde 9 , dont une, en particulier, refait surface dans la mémoire de Joyce au moment de sa fugue alors qu'elle hésite à aborder le camionneur qui lui permettra de quitter Sept-Îles. Si elle décide finalement de l'aborder, c'est qu'elle reconnaît en lui la figure d'un personnage russe qui était sur le timbre de la carte de l'oncle Jonas et qui représentait Vladimir Lénine :

L'anachronisme la fait sourire. Puis, elle redevient sérieuse. Si ce bougre d'oncle Jonas a eu le cran de fréquenter les quais verglacés de Leningrad à quatorze ans, qui pourra empêcher Joyce - tout aussi Doucet que lui — d'en faire autant? (p. 79)

Ensuite, la vérité au sujet de sa mère qui a fui la maison familiale vers le continent, permet à Joyce d'envisager son propre départ vers le continent. À ces figures de fuite, d'errance et de vie illégitime, s'ajoute l'importante découverte, dans un journal, d'une lointaine cousine nommée Leslie Lynn Doucette qui est parvenue à mettre en place un des plus grands réseaux de piratage par téléphone de l'histoire des États-Unis :

[Leslie Lynn] Doucette aussi connue sous le pseudonyme de Kyrie, est accusée d'avoir piraté des messageries vocales informatiques afin d'y implanter des "lignes d'information ». On estime que ces lignes d'information permettaient à plus de 150 complices d'échanger des numéros de cartes de crédit et de cartes d'appels interurbains [...] Citoyenne canadienne, Doucette s'était réfugiée aux États-Unis en 1987 après avoir

\footnotetext{
9 « Cet oncle légendaire, âgé d'à peine quatorze ans, avait remonté le fleuve jusqu'à Montréal où il s'était embarqué sur un cargo en partance pour Madagascar — et on ne l'avait plus jamais revu. Sa famille recevait parfois d'illisibles cartes postales envoyées de tous les ports du monde, que le grand-père Lyzandre punaisait fièrement aux murs de la maison. » (Dickner, 2005, p. 61)
} 
subi une sentence pour une fraude similaire en territoire canadien. (p. 76)

À l'instar de Leslie Lynn Doucette, Joyce trouve le moyen de réactualiser la profession de pirate lorsqu'elle découvre, dans les ordures de Montréal, un ordinateur :

Ce monde ne ressemble plus à celui d'hier. Les caisses enregistreuses, les guichets automatiques, les transactions par cartes de crédit, les téléphones cellulaires... L'Amérique du Nord ne sera bientôt plus qu'une série de réseaux informatiques connectés les uns aux autres. Ceux qui sauront manœuvrer un ordinateur pourront tirer leur épingle du jeu. Les autres manqueront le bateau. (p. 112)

Bien que ses activités de pirate se produisent essentiellement dans le monde virtuel, Joyce doit cependant intégrer et s'approprier l'espace réel afin d'y trouver clairement ses repères. Elle le fait en traçant une carte de la ville qui lui permet d'y « naviguer » selon les besoins du moment :

Le centre-ville tient au complet dans son cerveau, soigneusement découpé en quadrants, zones et sous-secteurs. On ne pêche pas n'importe quoi, n'importe où, n'importe quand. La composition des déchets change non seulement d'une ruelle à l'autre, mais également selon les jours de la semaine, les saisons, les fluctuations de la bourse, la politique extérieure américaine. Pour Joyce, tout cela s'organise en une carte complexe. (p. 207)

Alors que Noah trace une carte de la ville fondée sur les cultures immigrantes, porteuses des accents d'un ailleurs qui s'enracine dans l'ici, Joyce fait de Montréal un espace nautique où elle navigue à la manière de ses ancêtres pirates. Comme pour ces derniers, cette navigation se fait à la recherche de trésors (cartes d'identité, cartes bancaires, matériel informatique) et dans un jeu de cache-cache avec les autorités. 
Aussi, lorsque la GRC débarque chez elle Joyce, comme les pirates, trouve-t-elle refuge sur une île des Caraïbes, chez la mère de Maelo.

La destinée des deux personnages permet donc, par la réactualisation de leurs origines dans l'espace continental, de retracer une cartographie de l'Amérique et, plus particulièrement, de la Franco-Amérique - par le lien explicite des personnages, d'une part, aux Métis de Louis Riel ainsi qu'aux Acadiens déportés ou en fuite et, d'autre part, par la figure de Montréal comme lieu de transit ouvert sur les Amériques, où semblent s'effriter les repères géographiques et temporels ayant traditionnellement servi à déterminer les espaces identitaires. De locale qu'elle semble d'abord être, l'identité des personnages s'ouvre sur un espace global qui l'inscrit dans un réseau de contacts où le soi, descendant de différents foyers de la Franco-Amérique, parvient à trouver une place à la fois unique et continentale, comme tend d'ailleurs à le démontrer la «continentalité » qu'Arizna associe à l'identité amérindienne ainsi que la présence partout en Amérique de tous ces Doucet à l'orthographe singulière :

selon les circonstances, les lieux ou les subtilités de la grammaire ambiante, on les nommât aussi Doucet, Doucett, Douchette, Douchet, Douchez, Douçoit, Duchette, Ducette, Dowcett, Dusett, Ducit ou Dousette. (p. 58)

Ce constat n'est évidemment pas sans rappeler les propos d'Éric Waddell et Dean Louder qui perçoivent la cartographie de la Franco-Amérique, dans tout ce qu'elle présente de paradoxes, comme empreinte d'une pluralité de foyers, souvent fondée sur un rapport de plus en plus individualiste au monde, tout en représentant une réalité de dimension continentale. Cette cartographie se construit également dans le dialogue avec 
l'«autre» immigrant qui devient, dans le nouvel espace identitaire, une figure familière pour le soi.

Afin de trouver, individuellement, leur voie/x dans l'espace américain, les personnages de Nikolski, issus des différentes souches francophones sur le continent, sont donc amenés à repenser, en se basant sur ce qu'ils dégagent de leurs observations personnelles, la cartographie spatiale et culturelle de leur espace identitaire. Toutefois, si, chez Waddell et Louder, l'acte de tracer la carte des foyers francophones en Amérique constitue une première étape dans la reconnaissance de cette réalité continentale, chez Dickner, c'est l'acte de narration, dont le travail est de superposer les récits et les cartes recomposées des personnages, qui permet la mise en réseau des destinées individuelles pour en faire un «nouveau grand récit» de l'espace identitaire franco-américain.

\section{Narrer le « nouveau grand récit» de la Franco-Amérique}

Bien qu'il n'apparaisse que sporadiquement dans le roman, le narrateur marque clairement sa présence en se posant d'emblée sous le signe du soi qui raconte. Il explique dans le premier chapitre, par exemple, comment il a vendu la maison de sa mère, après que celle-ci était décédée, comment il a retrouvé le «compas Nikolski », qui devient en quelque sorte son objet fétiche, et comment, finalement, il en vient à se consacrer entièrement à son travail à la librairie de livres usagés. Si, comme nous l'avons déjà souligné, la mort de la mère et l'image du « compas Nikolski » donnent en quelque sorte le ton au récit du parcours que suivent Noah et Joyce en marge 
des référents identificatoires traditionnels, le travail de libraire du narrateur sert, pour sa part, à rassembler le tout en faisant des différentes destinées individuelles la matière d'un récit plus large, de dimensions continentales, pour reprendre l'expression de Waddell et Louder, et de configuration pluraliste. En fait, la librairie représente, dans le discours du narrateur, « un de ces coins du cosmos où les humains ont depuis longtemps perdu le contrôle sur la matière » (Dickner, 2005, p. 22) et où il est le seul à savoir bien s'y retrouver. Cet espace, comme celui que parcourent Noah et Joyce, possède donc sa propre cartographie, dont le narrateur retrace la configuration :

Chaque étagère supporte trois épaisseurs de livres et les planchers disparaissent sous des douzaines de boîtes en carton entre lesquelles serpentent d'étroits sentiers aménagés pour la circulation des clients. Le moindre interstice est mis à profit : sous le percolateur, entre les meubles et les murs, à l'intérieur du réservoir de la toilette, sous l'escalier et jusque dans l'exiguïté poussiéreuse de l'entretoit. Notre système de classement est parsemé de microclimats, de frontières invisibles, de strates, de dépotoirs, d'enfers désordonnées, de vastes plaines sans points de repères apparents - complexe cartographie qui repose essentiellement sur la mémoire visuelle, une faculté sans laquelle on ne dure pas longtemps dans le métier. (p. 22)

Le narrateur précise que, à cet espace à la cartographie particulière, s'ajoute une perception trouble de la temporalité alors qu'il affirme "que divers avatars de notre bouquinerie coexistent simultanément dans une multitude d'époques différentes, séparés par de très minces ellipses» (p. 22-23). Ainsi, son travail de libraire consiste à naviguer dans cet espace "géographique» et dans cette temporalité trouble de la librairie afin d'en maintenir l'équilibre. Une tâche d'autant plus importante que c'est par ce travail qu'il parvient à établir des 
réseaux de contacts entre les différentes unités qui habitent la librairie, les livres pris dans leur individualité, et le monde extérieur, celui des clients en quête d'informations pouvant être utilisées en vue de la complétude de soi :

Chaque livre qui entre ici peut rencontrer son prochain lecteur à n'importe quel moment de l'histoire de la boutique, aussi bien dans le futur que dans le passé. Lorsqu'elle trie un nouvel arrivage de livres, madame Dubeau [la propriétaire] consulte sans cesse son Encyclopédie Lavoisier - une trentaine de cahiers où elle répertorie toutes les demandes spéciales des clients depuis février 1971 - afin de voir si quelqu'un n'aurait pas, dix ans plus tôt, désiré l'un des livres fraîchement débarqués. (p. 23)

L'image de l'Encyclopédie est importante puisqu'elle accentue la dimension symbolique qui inscrit le narrateur dans un rôle de passeur de sens; celui que les clients découvrent dans les livres qui leur sont réservés. Un rôle qu'il assume d'ailleurs parfaitement :

Notre librairie est, en somme, un univers entièrement composé et gouverné par les livres - et il me semblait tout naturel de m'y dissoudre totalement, de vouer mon destin aux milliers de destins dûment empilés sur ces centaines d'étagères. (p. 24)

De la même manière qu'il se dissout dans l'espace de la librairie, le narrateur pénètre le récit qu'il fait de la destinée entrecroisée de Noah et de Joyce tout en occupant, par l'acte de narration, le même rôle de passeur.

Certes, à la fin du premier chapitre, qu'il présente comme un «prologue », le narrateur dit s'effacer de son récit pour ne narrer que les événements. Or, cette absence du narrateur en «je» ne signifie pas son effacement complet du récit; au contraire, le narrateur intervient régulièrement en commentant la perception qu'ont les personnages du monde qui les entoure. 
On n'a qu'à penser à cet exemple déjà cité où la présence d'une voiture sur la plaine laisse à Noah l'impression que la route passe au centre de sa salle de bain et que le narrateur constate que « cette image n'est pas très loin de la réalité » (p. 28); ou au commentaire qu'il fait au sujet de l'enthousiasme que Noah ressent par rapport à ses origines acadiennes: "Son enthousiasme reposait toutefois sur une erreur de perception, car en réalité ses ancêtres n'avaient pas été déportés. » (p. 3031); ou, encore, au supplément d'informations qu'il donne souvent afin de compléter la pensée des personnages, comme lorsque Maelo signale à Joyce que la poissonnerie Shanahan n'est pas au Japon :

Car chacun sait que les Nippons ont le cœur bien accroché, le regard d'acier, et qu'ils achètent les thons à l'encan, directement sur les quais sanguinolents. La clientèle de la Poissonnerie Shanahan est, comment dire, plus délicate [...]. (p. 98)

Aussi cette présence constante du narrateur permet-elle de repenser les liens entre les personnages comme étant des liens qui ont moins à voir avec des coïncidences ${ }^{10}$ qu'avec une stratégie narrative visant à tisser, à travers les "micro-récits » que représente le parcours des personnages, un réseau de communications qui permet une reconfiguration des «microrécits » en un « nouveau grand récit».

\footnotetext{
10 Les personnages sont membres de la même famille; ils habitent le même quartier; ils rencontrent les mêmes personnages à des moments différents, comme Maelo, le professeur Thomas Saint-Laurent, Arizna ou, encore, l'itinérant qui porte une tuque des Maple Leafs; ils lisent les mêmes livres. On pourrait nommer d'autres coïncidences qui servent à tisser, au fil du roman, un univers commun à travers lequel progressent les différents personnages. À ce sujet, voir l'article de Christine Otis (2010).
} 
Pourtant, ces interventions peuvent sembler troublantes parce que l'apparition du « je » se fait souvent dans l'ignorance des événements que vivent Noah et Joyce, créant un fossé entre les deux modes de narration, entre le récit du soi et celui des autres. Par exemple, lorsque Joyce visite le narrateur pour lui emprunter un livre, ce dernier, qui affirme ne connaître rien d'elle, a l'impression de reconnaître son « double féminin, une sorte de cousine débarquée de nulle part » (p. 277), et lorsqu'il rencontre Noah, à la fin du roman, il ne reconnaît pas celui qui, dès le début du roman, se présente comme son demi-frère. Ce jeu narratif s'explique en partie par la présence d'un livre qui traverse le roman et qui constitue une mise en abyme du roman de Dickner. Il s'agit en fait du «Livre sans visage », celui avec lequel Noah apprend à lire, qui deviendra, dans les mains du narrateur « je », le « Livre à trois têtes ». Déjà, dans les passages où Noah, au début du roman, et le narrateur, à la fin, nomment le livre, il est possible de retracer l'évolution de la quête identitaire qui unit les personnages. C'est grâce au narrateur que ce que Noah définit comme «une sorte de recueil d'histoires de marins » (p. 38) retrouve sa véritable identité :

pages 27 à 53 : une très ancienne monographie sur les îles aux trésors;

pages 71 à 102 : un traité vaguement historique sur les pirates des Caraïbes;

pages 37 à 61 : une biographie d'Alexander Selcraig, naufragé sur une île déserte. (p. 175)

Trois livres qui existent donc, à l'origine, individuellement, mais que la reliure rassemble sous une destinée commune :

Personne ne pouvait imaginer la trajectoire de ce livre. Après plusieurs décennies sur les rayons de la bibliothèque de l'université de Liverpool, il avait été volé par un étudiant, avait 
circulé de main en main, avait échappé à deux incendies puis, abandonné à lui-même, était retourné à l'état sauvage. Il avait parcouru des milliers de kilomètres dans plusieurs sacs, voyagé à fond de cale dans des caisses humides, avait été jeté pardessus bord, puis avait cheminé dans l'estomac acide d'une baleine avant d'être recraché et repêché par un scaphandrier analphabète. Jonas Doucet l'avait finalement gagné au poker dans un troquet de Tel-Aviv, un soir de bamboche. (p. 37)

Un parcours qui ne s'arrête pas là puisque, comme le résume Christine Otis, le livre, avant de trouver son identité, traverse le récit en passant de mains en mains :

[...] il se trouve en la possession de Noah, pour lui être ensuite volé ou emprunté par son amie Arizna, qui l'oublie ou le laisse volontairement à la librairie du narrateur; celui-ci le retrouve quelques années plus tard dans son sous-sol alors qu'il est en compagnie de Joyce, avant que Noah ne retombe lui-même dessus à la librairie et remette au narrateur la carte qui s'en était détachée et qui était toujours en sa possession. (2010)

Comme le roman de Dickner, le "Livre à trois têtes » semble trouver son sens dans la mise en contact de destinées singulières pour créer un «grand récit » unique :

ce sont des fragments au sens propre. Des débris, des déchets. Un relieur a récupéré les carcasses de trois livres et les a cousus ensemble. C'est une pièce d'artisans, pas un objet imprimé en série. (Dickner, 2005, p. 320)

$\mathrm{Si}$, à la fin du roman, il semble possible de poser la librairie comme une représentation de l'espace identificatoire dans lequel s'inscrivent les parcours de Noah et de Joyce, et le « Livre à trois têtes » comme une mise en abyme du procédé narratif menant à l'affirmation des identités, il semble également pertinent de poser l'ensemble du récit que fait le narrateur comme un espace de questionnement identitaire du soi. Devenir soi avec les autres, dans Nikolski, c'est en quelque sorte faire le récit des autres afin de 
découvrir sa propre voie/x dans la vie. En fait, il n'est pas étonnant que la rencontre entre le narrateur et ses personnages - au sens où il $\mathrm{y}$ a un véritable échange qui va au-delà du rapport froid de client à libraire qu'il a d'abord avec Joyce — ne se produise qu'à la fin du roman; parce qu'ils ont complété leur propre cheminement, Noah et Joyce semblent effectivement à même d'aider le narrateur à compléter le processus de libération déclenché à la mort de sa mère. Joyce, à la fin du roman, est celle qui achève le processus en brisant le «compas Nikolski» alors que Noah apporte au narrateur une complétude de soi en lui donnant le dernier morceau de son puzzle, c'est-à-dire en complétant l'univers hétéroclite du « Livre à trois têtes » :

J'approche la carte du Livre à trois têtes, comme la dernière pièce d'un casse-tête. Mon intuition s'avère exacte : la déchirure s'ajuste parfaitement à la reliure! Cette carte a donc été arrachée du livre il y a plusieurs années de cela... Je considère ce drôle de puzzle, médusé. Voilà une découverte qui contribue à obscurcir la question plutôt qu'à l'éclaircir. (p. 322)

Et si la découverte vient obscurcir la question, c'est que la fin d'un récit appelle toujours le début d'un autre, voire d'un récit autre, et que le narrateur, comme le livre, mais également comme Noah et Joyce, peut poursuivre sa route vers de nouvelles rencontres : "Je souris, hausse les épaules et, après avoir recollé la carte des Caraïbes à sa place, remets le Livre à trois têtes dans la boîte de liquidation. » (p. 321)

\section{Conclusion}

Le roman de Nicolas Dickner, on le constate, s'inscrit bien dans ce mouvement de dépassement identitaire qu'on retrouve dans 
le roman de la route québécois en proposant une lecture du monde qui, dans la construction de la cartographie de la FrancoAmérique, rassemble des histoires singulières qui ont marqué le continent. Une importance particulière est cependant accordée aux origines multiples des trois personnages, qui renvoient à l'histoire trouble de certains foyers fondateurs de la francophonie nord-américaine soit québécois, acadiens ainsi que métis et francophones de l'ouest. Ces origines que portent les personnages ne s'imposent pas comme totalité identitaire et permettent, justement parce qu'elles renvoient à des histoires troubles se reflétant sur l'ensemble de la Franco-Amérique, une construction identitaire non plus en marge du monde, mais dans un dialogue constant avec les différentes figures d'altérité qui habitent le territoire. Aussi, comme l'annoncent Éric Waddell et Dean Louder, cette Franco-Amérique, qui s'ouvre sur l'ensemble du continent, se dessine-t-elle à Montréal dans un dialogue constant avec les autres cultures, particulièrement sud-américaines, en les posant littéralement au centre même du processus d'identification des personnages. Ce qui donne à Nikolski un intérêt supplémentaire dans la constitution de ce «nouveau grand récit» continental de la présence francophone, c'est finalement le fait que ce récit ne nie pas l'histoire ancienne en effaçant les mythes de la fondation du territoire; au contraire, le roman de Dickner propose une réhabilitation de l'histoire continentale à travers des lectures individuelles qui permettent aux personnages d'y trouver une place qui leur est propre. La différence de cette histoire, prise dans la longue durée, en lien avec l'historiographie traditionnelle, tient au fait qu'elle ne représente pas, dans la perception des personnages, un récit linéaire et unique à une géographie, mais renvoie plutôt à une temporalité malléable et ouverte sur le continent. C'est grâce à cette malléabilité du temps historique et à 
cette absence marquée des frontières qui s'y rattachent traditionnellement qu'il devient possible de réactualiser, sans sombrer dans une continuité aveugle, les traces nécessaires à l'identification de soi. Cette nouvelle perception des repères spatiaux et temporels de l'identité du soi permet de sortir d'un cadre identitaire unique et de trouver, dans le récit des autres, un nouveau sens à la cartographie du soi. Ainsi, il semble possible d'affirmer que la société que décrit Dickner ne s'inscrit pas dans le schéma identitaire relevé par Józef Kwaterko dans l'évolution du roman québécois, mais qu'elle le dépasse, en quelque sorte, en proposant un récit qui ne fait pas de différence entre la « société pensée » et la "société vécue », un récit, en fait, qui permet aux deux modèles de cohabiter.

\section{Bibliographie}

Angenot, Marc (1978), «Le paradigme absent. Éléments d'une sémiotique de la science-fiction », Poétiques, n 33 , p. 74-89.

BOUCHARD, Michel (2001), «Les Lamoureux de l'Alberta: un parcours vaste et complexe», dans Dean Louder, Jean Morisset et Éric Waddell (dir.), Vision et visages de la FrancoAmérique, Québec, Septentrion, p. 31-45.

DiCKNER, Nicolas (2005), Nikolski, Québec, Alto.

HotTE, Lucie (2009), «La mémoire des lieux et l'identité collective en littérature franco-ontarienne», dans Anne Gilbert, Michel Bock et Joseph Yvon Thériault (dir.), Entre lieux et mémoire. L'inscription de la francophonie canadienne dans la durée, Ottawa, Presses de l'Université d'Ottawa, coll. «Amérique française », p. 337-367. 
KWATERKO, Józef (2001), «Clivages, ex-centricité, nomadisme : l'identité culturelle et l'imaginaire de l'espace dans le roman québécois », dans Jaap Lintvelt et François Paré (dir.), Frontières flottantes. Lieu et espace dans les cultures francophones du Canada/Shifting Boundaries. Place and space in the francophone cultures of Canada, Amsterdam/New York, Rodopi, p. 147-159.

MORENCY, Jean, Jeanette DEN TOONDER et Jaap LINTVELT (dir.) (2006), "Introduction », dans Romans de la route et voyages identitaires, Québec, Nota bene, coll. « Terre américaine », p. 5-13.

MORENCY, Jean (2008), « Dérives spatiales et mouvances langagières : les romanciers contemporains et l'Amérique canadiennefrançaise », Francophonies d'Amérique, n 26, p. 27-39.

- (2009), «Romanciers du Canada français: Gabrielle Roy, Jacques Poulin, Michel Tremblay, Roch Carrier », dans Lucie Hotte et Guy Poirier (dir.), Habiter la distance. Études en marge de La distance habitée, Sudbury, Prise de parole, coll. « Agora », p. 147-163.

MorisSET, Jean et Éric WADDELl (2000), Amériques, Montréal, L'Hexagone, coll. «Itinéraires ».

OTIS, Christine (2010), «Le jeu des coïncidences: une vraisemblance à construire. Les exemples de Nikolski de Nicolas Dickner et de La Kermesse de Daniel Poliquin ", Temps zéro. Revue d'étude des écritures contemporaines, $\mathrm{n}^{\circ} 2$, URL :http://tempszero.contemporain.info/document398.

Poulin, Jacques (1989 [1984]), Volkswagen blues, Montréal, Québec/Amériques, coll. "Littérature d'Amérique ».

WADDELL, Éric et Dean Louder (2008), «Conceptualiser et cartographier la Franco-Amérique : une tâche redoutable ", dans Dean Louder et Éric Waddell (dir.), Franco-Amérique, Québec, Septentrion, p. 13-23. 


\title{
Résumé
}

Le roman Nikolski de Nicolas Dickner met en scène la destinée croisée de trois personnages qui, ne se reconnaissant pas dans les espaces traditionnels qu'ils habitent, en viennent à reconfigurer la cartographie de leur appartenance intime au continent américain. Cette reconfiguration ne semble cependant possible que par la réactualisation d'un discours de l'origine puisé dans un passé marginalisé de la Franco-Amérique : c'est effectivement à travers les figures du Métis au territoire perdu et de l'Acadien devenu pirate dans la foulée du Grand Dérangement que les personnages se reconnaîtront. Ce sont ces figures singulières, réactivées par les personnages, qui constituent les balises de la nouvelle cartographie identitaire plurielle que trace, à travers une série de chassés-croisés, la narration de Nikolski.

\begin{abstract}
Nicolas Dickner's novel Nikolski presents the shared destinies of three characters who, because they do not recognize themselves in the traditional places they inhabit, end up reconfiguring the cartography of their intimate belonging to the American continent. This reconfiguration, however, appears only possible through the reactualization of a discourse of the origins that draws on a marginalized past of Franco America. Indeed, it is only thanks to the figures of the Métis carving out his existence in a lost territory and the Acadian turning into a pirate on the heels of the Grand Dérangement that the characters will find their bearings. These singular figures, who the characters bring to life, constitute beacons in a new, plural cartography of identity that is mapped out by Nikolski's narration through a series of to-ings and fro-ings.
\end{abstract}

